\title{
Adelheid Schellmann
}

\section{Der Breslauer Signator Virgilius Haugk: Komponist und Musiklehrer im Zeichen der Reformation}

Der vorliegende Beitrag befasst sich mit einem schlesischen Thema und stellt einen Breslauer Komponisten aus der Mitte des I6. Jahrhunderts in den Fokus: Virgilius Haugk. Er ist einer der zahlreichen, heute kaum noch bekannten sogenannten »Kleinmeister« der Reformationszeit - wobei dieser Terminus ganz im Sinne Wolfram Steudes verstanden werden soll:

»Der Begriff >Kleinmeister wird hier mit vollem Bedacht angewandt. Ebensowenig wie in der Kunstwissenschaft soll er auch hier abwertende Bedeutung haben, sondern lediglich einen gewissen Abstand kleinerer Komponisten zu ihren die Maßstäbe setzenden großen Zeitgenossen anzeigen. Daß sich das künstlerische Durchschnittsniveau der Zeit im Werk der Kleinmeister repräsentiert, macht nicht zuletzt auch ihren Wert für die Geschichtsschreibung aus. Neuland haben sie im allgemeinen nicht erschlossen, sie sind aber den Bahnbrechern auf dem Fuße gefolgt. $\aleph^{1}$

Für die stilistische Einordnung von Haugks musikalischen Werken wird immer wieder ein Zitat aus seinem musiktheoretischen Lehrwerk herangezogen, den Erotemata musicae practicae ad captum puerilem formata (Breslau: Andreas Winkler I54I). Am Ende des Traktats bezeichnet Haugk Josquin Desprez als »musicus sine controversia excellentissimus «. In Anlehnung daran glaubte etwa Wilfried Brennecke in Haugks Kompositionen zwei Vorbilder erkennen zu können, nämlich den Stil Thomas Stoltzers und den niederländischen Josquin-Stil. ${ }^{2}$ Doch ist diese Verortung Haugks zwischen zwei Personalstilen problematisch, weil sie nur sehr schwer - wenn überhaupt - analytisch überzeugend nachgewiesen werden kann. Völlig abgesehen davon ist eine derartige Josquin-Bewunderung in der fraglichen Zeit schlicht als Topos zu betrachten.

I Wolfram Steude, Untersuchungen zur mitteldeutschen Musiküberlieferung und Musikpflege im I6. Jahrbundert, Leipzig 1978 (Musikwissenschaftliche Studienbibliothek Peters), hier S. 9.

2 Wilfried Brennecke: Art. »Haugk, Virgilius«, in: $M G G$ Bd. 5, Kassel und Basel 1956, Sp. I826-I827. 
Im folgenden soll Virgilius Haugks »Bedeutung für die Geschichtsschreibung « herausgearbeitet und dargestellt werden, dafür spielen seine Biographie, sein Umfeld und seine überlieferten Werke die wichtigsten Rollen.

II.

Über die Biographie des Virgilius Haugk weiß man nicht viel: ${ }^{3}$ Geboren wurde er zwischen I490 und I500 in Böhmen. Wo Haugk seine Jugend verbrachte und welche Ausbildung er genoss, ist nicht bekannt. Bis 1522 lebte er im schlesischen Neisse (poln. Nysa), wo er Pfründeneinkünfte bezog und wo ein gewisser Silvester Haugk, wahrscheinlich sein Bruder, über mehrere Jahre hinweg als Kantor tätig war. Zwischen 1538 und I544 lässt sich Virgilius Haugk dann als Signator an der Stadtpfarrschule St. Elisabeth in Breslau nachweisen. Außerdem ist seine Heirat im Jahr I542 belegt. Aus dem Testament der Ehefrau von I555 wiederum geht hervor, dass ihr Mann zu dieser Zeit nicht mehr lebte. Außer diesen Daten gibt Betty Karol Fairchilds Wilson die Information, dass Haugk I55I an der Wittenberger Universität immatrikuliert war. ${ }^{4}$ Das ließ sich jedoch in der Wittenberger Universitätsmatrikel nicht verifizieren. ${ }^{5}$

Die Überlieferungssituation von Haugks Kompositionen stellt sich ebenfalls nicht besonders üppig dar:

\section{Drucke}

Sacrorum hymnorum liber primus, Wittenberg I542 (RISM I542 ${ }^{12}$ )

- Ad cenam agni providi à 4 (Ostern)

3 Haugks Biographie ist skizziert in den entsprechenden Artikeln in $M G G, M G G^{2}$ und NewGrove ${ }^{2}$ (Brennecke, Art. »Haugk, Virgilius« (s. Anm. 2).; Rainer Birkendorf, Art. »Haugk, Virgilius«, in: $M G G^{2}$, Personenteil Bd. 8, Kassel u.a. 2002, Sp. 868-869; Wilfried Brennecke: Art. »Haugk, Virgilius", in: New Grove ${ }^{2}$, Bd. II, London 200I, S. I37.) Ausführlicher und mit Quellenbelegen versehen findet sie sich bei Gustav Bauch, Geschichte des Breslauer Schulwesens in der Zeit der Reformation, Breslau I9II (Codex Diplomaticus Silesiae 26), hier S. Iogf und aktueller bei Klaus Wolfgang Niemöller, Untersuchungen zu Musikpflege und Musikunterricht an den deutschen Lateinschulen vom ausgehenden Mittelalter bis um 1600, Regensburg 1969 (Kölner Beiträge zur Musikforschung 54), hier S. 9. Außerdem findet Haugk in den folgenden Lexika Erwähnung: Ernst Ludwig Gerber, Nenes historisch-biographisches Lexikon der Tonkünstler (I8I2-I8I4), Bd. 2: Teil I-2 (I8I2), hrsg. von Othmar Wessely, Nachdruck Graz 1966, Sp. 52I und Gottfried Johann Dlabačz, Allgemeines historisches Künstlerlexikon für Böhmen und zum Theil auch für Mähren und Schlesien. Drei Bände in einem Band, Nachdruck der Ausgabe Prag I8I5 und Prag I9I3, Hildesheim u.a. I973, Sp. 577.

4 Betty Karol Fairchilds Wilson, Choral pedagogy. Crossroads of theory and practice in sixteenth century Germany Ph.D. diss. Boston I995, hier S. 527.

5 Im Juli I553 immatrikulierte sich ein »Joannes Haug Cziecensis« in Wittenberg, zu dieser Zeit wäre Virgilius Haugk etwa 60 Jahre alt gewesen. Karl Eduard Förstemann, Album Academiae Vitebergensis I502 - I602, Leipzig I84I, hier S. 283. 
- Accende lumen sensibus à 4 (Pfingsten $)^{6}$

- Ogloriosa Domina à 4 (Mariä Himmelfahrt)

- Angelum pacis Michael à 4 (Michaelis) ${ }^{7}$

Newe Deudsche Gesenge, Wittenberg I544 (RISM I544 ${ }^{21}$ )

- Wir gläuben all an einen Gott à 5 (Nr. 42)

\section{Handschriften}

Ms.mus. Bártfa 22 (nur Tenor und Vagans erhalten $)^{8}$

- Ubi caritas et amor à 5 (Nr. II6)

Ms.mus. Bártfa 23 (nur Bassus erhalten)

- Pater, filius, sanctus spiritus à 5 (Nr. II2)

Utrechter Liber Psalmorum (nur Discantus erhalten) ${ }^{9}$

- Deus quis similis erit tibi (Ps. 82; Nr. 22)

Zwickau Ratsschulbibliothek Mus.Ms. $73^{10}$

- Verbum caro factum est à 4 (Weihnachten)

6 Die beiden Hymnen Ad cenam agni providi und Accende lumen sensibus finden sich außerdem in der Handschrift Cod.mus.fol. I 24 der Württembergischen Landesbibliothek Stuttgart. Diese Handschrift stammt aus dem Bestand der ehemaligen Stuttgarter Hofkapelle und enthält vor allem Abschriften aus Rhaws Sacrorum hymnorum liber primus. Siehe dazu Clytus Gottwald, Die Handschriften der Württembergischen Landesbibliothek Stuttgart. Erste Reibe, Erster Band: Codices Musici (Cod. Mus. fol. I I-7I), Wiesbaden I964, hier S. 43-46.

7 Auch Angelum pacis Michael findet sich in einer Parallelüberlieferung, und zwar im handschriftlichen Zusatz zu Mus.pr. Bártfa 6. Hier handelt es sich ebenfalls in weiten Teilen um eine Abschrift aus Rhaws Sacrorum hymnorum liber primus. Siehe dazu Róbert Árpád Murányi, Thematisches Verzeichnis der Musiksammlung von Bartfeld (Bártfa), Bonn I99I (Deutsche Musik im Osten 2), S. XXXI (Nr. 6), S. I65 (Nr. 2422) und S. 440 (Nr. 2422).

8 Die gesamte in Budapest aufbewahrte Bartfeld-Sammlung ist verzeichnet bei Murányi, Thematisches Verzeichnis (s. Anm. 8). Zu Ms.mus. Bártfa 22 und 23 siehe außerdem Hans Albrecht, "Zwei Quellen zur deutschen Musikgeschichte der Reformationszeit. Die Handschriften Bártfa 22 und 23 des Ungarischen Nationalmuseums zu Budapest «, in: Die Musikforschung I (1948), S. 242-285, zu Haugk dort S. 253, 265 und 278.

9 Willem Elders, »Ein handschriftlicher >Liber Psalmorum < aus der deutschen Überlieferung «, in Formen und Probleme der Überlieferung mehrstimmiger Musik im Zeitalter Josquins Desprez, hrsg. von Ludwig Finscher, München I98I (=Wolfenbütteler Forschungen 6), S. 47-69. Haugks Vertonung wird dort nur im Inhaltsverzeichnis der Handschrift erwähnt (S. 60). Siehe auch Martin Just, »Die Schalreuter-Handschrift ZwickauR 73. Ein vorläufiger Bericht«, in: Gestalt und Ent stehung musikalischer Quellen im I5. und I6. Jahrbundert, hrsg. von Martin Staehelin, Wiesbaden I998, S. I79-I92, hier v. a. S. I8I-I84. Wolfram Steude kannte das Stimmbuch noch nicht. Haugks Psalmvertonung fehlt in der Aufzählung seiner Werke im $M G G^{2}$-Artikel (Birkendorf: Art. »Haugk, Virgilius«, s. Anm. 3).

IO Nachweis bei Reinhard Vollhardt, Bibliographie der Musik-Werke in der Ratsschulbibliothek zu Zwickau, Leipzig I893-I896 (Beilage zu den Monatsheften für Musikgeschichte), S. 4-I4; moderne Edition der Handschrift: Die Handschrift des Jodocus Schalreuter (Ratsschulbibliothek Zwickau 
- Ite in orbem à 5 (Christi Himmelfahrt)

- Apparuerunt apostolis à 5 (Pfingsten)

- In monte oliveti à 5 (Gründonnerstag)

Auffällig ist die Tatsache, dass alle Quellen, in denen Werke von Haugk überliefert sind, einen Bezug zu Wittenberg und zu Georg Rhaw haben. Bei den beiden Drucken aus Rhaws Offizin ist das offensichtlich. Für die Handschriften soll der Zusammenhang kurz erläutert werden: Die beiden Bártfa-Handschriften stammen aus dem Bestand der evangelischen Stadtpfarrkirche St. Aegidi im oberungarischen Bártfa (Bartfeld, slowak. Bardejov). ${ }^{11}$ Oberungarn ist der nordöstliche Teil des habsburgischen Ungarn, der nach der Schlacht von Mohács I526 und dem Fall von Buda I54I nicht von den Osmanen besetzt worden war, und gehört heute im wesentlichen zur Slowakei. In der Mitte des I6. Jahrhunderts setzte sich dort in mehreren Städten die Reformation durch. Zeugnis dafür sind drei von Philipp Melanchthon und der Confessio Augustana beeinflusste Bekenntnisschriften, deren älteste den Titel Confessio Pentapolitana trägt. ${ }^{2}$ Fünf oberungarische königliche Freistädte nahmen I549 die Pentapolitana an, neben Bartfeld waren dies Eperies (ungar. Eperjes/slowak. Prešov), Klein-Zeben (Kisszeben/Sabinov), Kaschau (Kassa/Košice) und Leutschau (Lő́cse/Levoča).

Der wohl maßgebliche Verfasser der Pentapolitana ist auch der Schlüssel für die engen Beziehungen zwischen Bartfeld und Wittenberg: Leonhard Stöckel (I5IO-I560) aus Bartfeld studierte ab dem Wintersemester I530/3I zunächst bis I533/34 in Wittenberg, bekleidete zwischen I534 und I536 das Amt des Rektors an der Schule in Eisleben, und kehrte dann für weitere drei Jahre nach Wittenberg zurück. ${ }^{13}$ I538 berief der Rat der Stadt Bartfeld Stöckel an die dortige Schule

Mus.Ms. 73), hrsg. von Martin Just und Bettina Schwemer, 4 Bände, Wiesbaden u.a. 20042006 (Das Erbe deutscher Musik II5a-II6b). Siehe außerdem Just, »Die Schalreuter-Handschrift ZwickauR 73" (s. Anm. 9) und Bettina Schwemer, Mehrstimmige Responsorienvertonungen in deutschen Quellen des Is. und 16. Jahrbunderts, 2 Bände, Augsburg 1998 (Collectanea Musicologica 8/I-II), hier Bd. I, S. 24-26.

I I Steude, Untersuchungen (s. Anm. I), hier S. 19-23. Zur Geschichte Bartfelds im I6. und 17. Jahrhundert und zur Musikpflege in dieser Zeit siehe Murányi, Thematisches Verzeichnis (s. Anm. 8), hier S. VII-XIX.

I 2 Max Josef Suda, »Der Einfluß Philipp Melanchthons auf die Bekenntnisbildung in Oberungarn (Confessio Pentapolitana, Confessio Heptapolitana und Confessio Scepusiana)«, in: Melanchthon und Europa, Bd. I: Skandinavien und Mittelosteuropa, hrsg. von Günter Frank und Martin Treu, Stuttgart 200I (=Melanchthon-Schriften der Stadt Bretten 6,I), S. I85-20I. Hintergrund für die Entstehung der oberungarischen Bekenntnisschriften ist, dass der ungarische König Ferdinand (der spätere Kaiser Ferdinand I.) die Confessio Augustana als innere Angelegenheit des Reiches betrachtete, die für Ungarn keine unmittelbare Gültigkeit habe (ebenda, S. I86).

I 3 Eine ausführliche Darstellung der Biographie Leonhard Stöckels bietet Otto Clemen als Kommentar zu einem Brief Luthers an den Rat der Stadt Bartfeld vom 17. April I539: D. Martin Luthers Werke. Kritische Gesamtausgabe. [Abteilung 4:] Briefwechsel, Bd. 8: I537-I539, hrsg. von Otto Clemen, 
zurück, doch auf Bestreben Melanchthons blieb Stöckel noch ein Jahr in Wittenberg. Auch nach Stöckels Rückkehr nach Bartfeld versuchte Melanchthon immer wieder, ihn von dort an einen anderen Ort zu holen, neben dem Argument der "Türkengefahr« brachte er I544 einen Ruf auf die Pfarrstelle in Mansfeld ins Spiel, dem Stöckel jedoch ebenso wenig folgte wie dem Ruf auf eine Rektorenstelle in Breslau. Stattdessen blieb er bis zu seinem Tod 1560 in Bartfeld und wurde zu einer entscheidenden Figur der Reformation in Oberungarn.

Stöckels hervorragende Beziehungen nach Wittenberg blieben nicht ohne Folge: Wie Wolfram Steude nachgewiesen hat, waren zwischen I53I und I6O2 insgesamt 24 Bartfelder Studenten in Wittenberg immatrikuliert (unter ihnen fünf Mitglieder der Familie Stöckel), die regelmäßig Musikdrucke und -handschriften in ihre Heimat mitbrachten. Diese Musikalien mit Wittenberger Provenienz - so folgert Steude - machen den größten Teil der Musiksammlung von Bártfa aus.

Die beiden Handschriften Ms.mus. Bártfa 22 und 23 sind außerdem Teil einer Gruppe von sieben Handschriften, die Steude in ihrer Konstellation identifiziert und einem Wittenberger Hauptschreiber (womöglich Lazarus Enderlein) zugeordnet hat. ${ }^{14}$ Neben den beiden Bartfeld-Handschriften zählen zu der Gruppe die Sammlungen Dresden, Sächsische Landesbibliothek Mus. 1-D-3 und Mus. 1D-4, sowie Zwickau, Ratsschulbibliothek Mus.Ms. 8I,2, Mus.Ms. IO०,4 und Mus.Ms. I06,5. Steude betrachtet sie als Druckvorlagen für geplante Publikationen Rhaws, die aus nicht bekannten Gründen nach dessen Tod 548 von seinen Erben nicht mehr verwirklicht worden sind.

Die anderen beiden Quellen, der Utrechter Liber Psalmorum und die Zwickauer Stimmbücher Mus.Ms. 73, stammen ebenfalls von einer Schreiberhand, und zwar von der des Zwickauer Cantors Jodocus Schalreuter (I486/87-I55O). Schalreuter hatte an der Universität Leipzig studiert und wurde I525 Bürger der Stadt Zwickau. ${ }^{15}$ Dort bekleidete er wohl ab Mitte oder Ende der I520er Jahre das Amt des Kantors an der Ratsschule. Nachdem Zwickau im Schmalkaldischen Krieg an den albertinischen Herzog Moritz von Sachsen gefallen war, wurde Schalreuter wegen seiner Treue zum ernestinischen Kurfürsten Johann Friedrich der Stadt verwiesen. Seine Bemühungen um eine Wiederaufnahme scheiterten daran, dass er nicht bereit war, Moritz als neuen sächsischen Kurfürsten anzuerkennen. Schal-

Weimar 1938, hier S. 406-409. Weitere und neuere Literatur zu Stöckel listet Suda, »Einfluß « (s. Anm. I2), hier S. I89f, v. a. Anm. 5.

I4 Steude, Untersuchungen (s. Anm. I), hier S. I38-I44. Siehe auch Marie Schlüter, Musikgeschichte Wittenbergs im I6. Jahrhundert. Quellenkundliche und sozialgeschichtliche Untersuchungen, Göttingen 2010 (Abhandlungen zur Musikgeschichte I8), hier v. a. S. I28-I38.

I 5 Zu Schalreuters Biographie siehe Martin Just: Art. »Schalreuter, I. Jodocus«, in: $M G G^{2}$, Personenteil Bd. I4, Kassel u.a. 2005, Sp. II83-II85 sowie Die Handschrift des Jodocus Schalreuter (s. Anm. IO), hier Bd. I, S. VII-XI und Otto Clemen, »Jodocus und Paul Schalreuter in Zwickau ", in: Zeitschrift für Musikwissenschaft I5 (1932/33), S. 320-323. 
reuters Widerstand gegen Moritz und das Augsburger Interim gingen so weit, dass er sich I550 aktiv an der Verteidigung Magdeburgs gegen die Verfechter des Interims beteiligte und dieses Engagement mit seinem Leben bezahlte.

Die musikalische Kompetenz Jodocus Schalreuters war zu seinen Lebzeiten besonders im Wittenberger Umfeld bekannt und geschätzt, wie Dokumente von Philipp Melanchthon, Antonius Musa und Georg Rhaw belegen. ${ }^{16}$ Außerdem ist Schalreuter der Widmungsträger von Rhaws Officia Paschalia. De Resurrectione et Ascensione Domini, Wittenberg 1539 (RISM I539 ${ }^{14}$ ), nachdem er Rhaw bei der Auswahl der Stücke für den Band geholfen hatte. ${ }^{17}$

Musikgeschichtlich bedeutsam ist aus heutiger Perspektive vor allem die Zwickauer Schalreuter-Handschrift. Sie besteht aus sechs Stimmbüchern, und wurde von Schalreuter wahrscheinlich ab Mitte der I530er Jahre planvoll angelegt. ${ }^{18}$ Gegliedert ist die Handschrift in zweimal drei Abteilungen, nämlich Psalmvertonungen und Responsorien, jeweils zu vier, fünf und sechs Stimmen. Innerhalb dieser Abteilungen lässt sich allerdings keine liturgische Ordnung erkennen. Das spricht neben der sorgfältigen kalligraphischen Gestaltung dafür, dass Schalreuter die Handschrift nach Gelegenheit und für den privaten Gebrauch anfertigte (und nicht etwa als Druckvorlage für Rhaw). Außerdem finden sich alle Komponistenzuweisungen im Bassus-Stimmbuch und es ist bekannt, dass Schalreuter selbst Bass gesungen hat. Sein »eigenes« Stimmbuch hat er also mit den meisten Informationen versehen.

Der Utrechter Liber Psalmorum ist auch von Schalreuter zusammengestellt bzw. geschrieben worden und dürfte etwa gleich alt sein wie die Zwickauer Stimmbücher. ${ }^{19}$ Doch unterscheiden sich beide Handschriften vor allem in zwei wesentlichen Punkten: Erstens ist das Utrechter Stimmbuch nicht so sorgfältig angelegt wie die Zwickauer Handschrift. ${ }^{20}$ Zweitens weist der Liber Psalmorum eine deutlich klarere Ordnung auf als die einzelnen Abteilungen des Zwickauer Manuskriptes: Die in der Utrechter Handschrift enthaltenen Psalmen folgen von Nr. I bis 43 und von Nr. 45 bis 55 der Ordnung des Psalters. ${ }^{21}$

I6 Die meisten Briefe stammen aus dem Umfeld des Zwickauer Ratsherrn Stephan Roth, der in Wittenberg studiert hatte und dessen Frau eine Schwägerin Georg Rhaws war. Siehe dazu das Vorwort zu Die Handschrift des Jodocus Schalreuter (s. Anm. IO), hier Bd. I, S. IXf.

I 7 Ebenda, S. IX.

I 8 Zur Entstehungsgeschichte und der Gestaltung der Handschrift siehe ebenda S. XI-XIV, außerdem Schwemer, Mehrstimmige Responsorienvertonungen (s. Anm. IO), hier Bd. I, S. 24-26 und Just: »Die Schalreuter-Handschrift ZwickauR 73" (s. Anm. 9).

I9 Ebenda S. I8I. Willem Elders hatte noch die Vermutung geäußert, Schalreuter habe die Utrechter Sammlung erst in seinen beiden letzten Lebensjahren angelegt (Elders: »Ein handschriftlicher >Liber Psalmorum«« (s. Anm. 9), hier S. 49).

20 Für einen Schriftvergleich siehe die Abbildungen I und 2 ebenda S. $50 f$.

2 I Just, »Die Schalreuter-Handschrift ZwickauR 73«(s. Anm. 9), hier S. I82. 
Auch wenn die Utrechter und die Zwickauer Handschrift beide von Schalreuter geschrieben wurden, so kann doch allenfalls das Utrechter Stimmbuch als Druckvorlage für Rhaw gelten, die Zwickauer Handschrift war für den privaten Gebrauch gedacht.

Haugks Kompositionen sind also nur aus Quellen überliefert, für die sich eine Beziehung zu Wittenberg und zu Georg Rhaw nachweisen lässt. Ob diese Verbindung stilistische Konsequenzen hat, soll unten diskutiert werden.

\section{III.}

Zunächst verdient jedoch der Titel »Signator«, den Haugk trug, eine besondere Erwähnung: Bezeichnet wird damit ein Schul- und Kirchenmusiker - eine Besonderheit, die von Breslau ausging und bald in ganz Schlesien üblich war. ${ }^{22}$ Die Titel »Cantor« und »Succentor« waren als officium in ecclesia dem Prälaten des Domkapitels bzw. dem Vikar vorbehalten, und hatten - so die Formulierung Fritz Feldmanns - »mit der praktischen Musikausübung nichts zu tun. ${ }^{23}$ Die Schulmusiker der Breslauer Domschule und der beiden Stadtpfarrschulen St. Elisabeth und St. Maria Magdalena bezeichnete man hingegen als Signator und Subsignator.

Der Begriff »Signator « leitet sich von lat. signum ab und hängt wahrscheinlich mit der Tätigkeit des Signators im kirchlichen Bereich zusammen: Der Signator gab »die `Zeichen<, die mit der äußeren Ordnung des Chorgesanges wie die zum Einzug, zur Gruppierung usw. zusammenhingen. ${ }^{24}$ In der Schule war der Signator für den Musikunterricht zuständig, also das Einüben des gregorianischen Chorals für die Gottesdienste, außerdem für den Leseunterricht der elementarii.

Virgilius Haugk war zwar auf dem Papier ein solcher Signator, doch fällt genau in seine Amtszeit an der St. Elisabeth-Schule in Breslau eine Veränderung dieser Situation. I523 bekannte sich der Rat der Stadt Breslau offiziell zur Reformation und übernahm zwei Jahre später das Patronat über die beiden Pfarrschulen St. Elisabeth und St. Maria Magdalena. ${ }^{25}$ Die Schul- und Kirchenordnung von I528 hält

22 Siehe dazu vor allem Niemöller, Untersuchungen (s. Anm. 3), hier S. 3-IO und S. 628f., außerdem Bauch, Geschichte (s. Anm.3), hier S.340 und Fritz Feldmann, »Studien zur Geschichte der Schulmusik Schlesiens«, in: Hamburger Mittel-und Ostdeutsche Forschungen 7 (1970), S. I53-206, hier S. I65f.

23 Ebenda S. I65.

24 Niemöller, Untersuchungen zu Musikpflege und Musikunterricht an den deutschen Lateinschulen vom ausgehenden Mittelalter bis um I600 (s. Anm. 3), hier S. 628f.

25 Die Einführung der Reformation in Breslau wird festgemacht an der Berufung des Dr. Johann Heß zum Pastor der Maria Magdalenen-Kirche durch den Rat der Stadt und gegen den Widerstand des Domkapitels. Siehe dazu Günter Elze, Breslau. Biographie einer deutschen Stadt, Leer 1993, hier S. 43. Zur Biographie von Heß siehe Georg Kretschmar, Art. »Heß, Johann«, in: Neue Deutsche Biographie, Bd. 9, Berlin 1972, S. 7-8, außerdem Werner Bellardi, »Johann Heß «, in: Schlesische Lebensbilder 4 (I93I), S. 29-39. 
fest, worin die Aufgaben des Signators zu dieser Zeit bestanden. ${ }^{26}$ Darin bestimmt der Rat, »das in yder schule ein schulmeister sein soll, drei baccalaurien, ein signator und zwene auditores. ${ }^{27}$ Darauf folgen Ausführungen über die Pflichten und den Lohn der verschiedenen Lehrer. Über den Signator heißt es:

Der signator soll haben zu lohne ein jar aus sechs mark; dorvor soll her singen uber die wochen vesper und messe und am sontage und feiertagen, welche im von den pfarhern ernent, sol her singen metten, vesper und mess, doneben etlich antiphon und responsoria, aber sunst deutze lieder, die im angezeiget werden. ${ }^{28}$

Damit ist der Signator eindeutig als Kirchenmusiker beschrieben. Außerdem wird deutlich, dass die Breslauer Gemeinden nach der Reformation an zwei Horen des Stundengebets festhielten, während der Woche an der Vesper, an Feiertagen außerdem an der Mette bzw. Matutin. Indem in der Kirchenordnung von deutschen Liedern die Rede ist, wird wiederum klar, dass die anderen Gesänge lateinische Texte hatten. Deren Verwendung im protestantischen Gottesdienst ist in der Frühphase der Reformation häufig im Umfeld von Lateinschulen zu finden. ${ }^{29}$ Vesper und Mette waren dabei in erster Linie Schülergottesdienste und hatten deswegen ihren pädagogischen Zweck zu erfüllen: Die Schüler sollten sich im Singen und im Lateinischen üben und gleichzeitig mit den Bibeltexten vertraut werden.

Der Signator hatte außerdem weitere Pflichten, mit denen er sein Gehalt aufbessern konnte: ${ }^{30}$

Dieweil man im aber in den horis keine stelle geschicken kann, und die zwen auditores mit vil kleinen knaben uberleget, soll her in der schul die elementarios des tages etliche stunden noch befel der hie zuvordenten herrn doctor den zwehen auditoribus helfen uberlesen; und

26 Ein Abdruck dieser Schulordnung findet sich in: Die evangelischen Kirchenordnungen des XVI. Jahrhunderts Bd. 3: Die Mark Brandenburg. Die Markgrafenthïmer Oberlausitz und Niederlausitz, Schlesien, hrsg. von Emil Sehling, fortgeführt vom Institut für Evangelisches Kirchenrecht der Evangelischen Kirche in Deutschland zu Göttingen, Leipzig 1909, hier S. 396-400. Außerdem gibt es eine sprachlich modernisierte Fassung mit einigen Erläuterungen bei Bauch, Geschichte (s. Anm. 3), hier S. 72-77.

27 Die evangelischen Kirchenordnungen (s. Anm. 26), hier S. 396.

28 Ebenda S. 397.

29 Siehe dazu (wenn auch ohne Bezug zu Breslau) Jürgen Heidrich, »>Deutsch oder lateinisch nach bequemigkeit?< Zur Bedeutung der Volkssprache für die protestantische Vesperpraxis im I6. Jahrhundert ", in: Kirchenmusikalisches Jahrbuch 82 (1998), S. 7-20; Schwemer, Mehrstimmige Responsorienvertonungen (s. Anm. IO), hier Bd. I, S. I95-I98. Für Schlesien konstatiert Fritz Feldmann einen im Gegensatz zu anderen deutschen Territorien deutlich geringeren Eingriff in die alte Liturgie, den er mit den »maßvollen kirchlichen Reformen« sowie dem besonders stark ausgeprägten Lateinschulwesen erklärt; siehe Feldmann: »Studien« (s. Anm. 22), hier S. I66.

30 Im Gegensatz zum Schulmeister, der 40 Mark im Jahr verdiente, und den Baccalaurien, die je zwanzig Mark bekamen, war der Signator mit seinen sechs Mark deutlich schlechter besoldet. 
dorvor sol man im geben ein jor lang vier mark zu den obgenannten sechs marken $[\ldots]$

Doneben was im von dem begrebnuss der todten zustendig noch ausweisung der selben ordenung, mag her auch seinen vorgesatzten lohn unschedlich doneben nehmen. ${ }^{31}$

Auch durch dieses Dokument wird die kirchenmusikalische Funktion des Signators deutlich, denn neben den regulären Gottesdiensten war er auch für Begräbnisse zuständig. Außerdem wurde er als Unterstützung für die anditores beim Leseunterricht der Schulanfänger eingesetzt.

Auch der schulische Musik- und Gesangsunterricht zählte zu den Aufgaben des Signators:

Es sol auch den knaben alle wochen ein lection nach ordenung der herrn doctor in der musica gelesen, und im singen treulich und fleissig so wol als in andern genotigten kunsten underweist und geubet werden. ${ }^{32}$

Und schließlich war der Signator für die Disziplin der Chorknaben verantwortlich:

Am sonnobent, sontag und andern feiertagen, so man in der schulen nicht list, sollen alle knaben zur messe und zur vesper in den chor gehen, aldo singen, und nicht vor geordneter zeit aus dem chore laufen; dorauf soll der signator ein fleissig aufschauen haben, und so sie hiraus laufen, sie vermerken und dorum zimmlich zu seiner zeit strafen. $^{33}$

I540 wird in Breslau erstmals die Bezeichnung »Cantor« für das Amt des Schulund Kirchenmusikers verwendet - also mitten in der Amtszeit von Haugk (I538I544). Die Quelle dafür ist ein Memorialzettel, mit dem Ambrosius Moiban, der Pastor von St. Elisabeth und einer der beiden Verfasser der zitierten Schul- und Kirchenordnung von I528, den Rat der Stadt um Ergänzungen zu eben dieser Ordnung bittet. ${ }^{34}$

Im dritten Artikel bemerkt er, für Hochzeitsgottesdienste sei es nötig »das eine ordnung gemacht werde, was man dem schulmeister, dem musico oder cantori, den baccalaurien vnd den andern gesellen von solchem geprenge, so man das $\mathrm{Te}$

3 I Die evangelischen Kirchenordnungen (s. Anm. 26), hier S. 397.

32 Ebenda S. 398.

33 Ebenda.

34 Abgedruckt bei Gustav Bauch, Aktenstücke zur Geschichte des Breslauer Schulwesens im XVI. Jahrhundert. Evangelische Realschule II zu Breslau. Wissenschaftliche Beilage zum Jahresbericht I898, Breslau I898, hier S. 42f. Siehe auch Niemöller, Untersuchungen (s. Anm. 3), hier S. 7. 
deum laudamus singet und figurirt, geben sol, auch so es der signator schlecht [= schlicht $]$ hinweg singet. ${ }^{35}$

Diese Textpassage benennt den Unterschied zwischen Cantor und Signator, der von der Mitte bis zum Ende des I6. Jahrhunderts in Breslau Bestand haben sollte: Der Cantor ist für den figurierten Gesang, der Signator dagegen für den schlichten Choralgesang zuständig. ${ }^{36}$ Der Memorialzettel nennt zwar zum ersten Mal den Titel Cantor, doch taucht dieser als offizielle Amtsbezeichnung in Breslau erst 1558 auf. ${ }^{37}$ Daneben blieb der Signatorentitel bis zum Ende des Jahrhunderts erhalten.

Das Verhandlungsergebnis zwischen Pfarrer und Rat von I540 zeigt, dass der Musikunterricht der Maria Magdalenen-Schule verbessert werden musste, um mit demjenigen an der Elisabeth-Schule mithalten zu können:

[...] wo ein gelerther musicus zw bekommen, das man yme das officium der cantorey eyngebe, dorczu auch jerlichen die Io cleine margk, domit er die jugenth in massen wie zw S. Elisabeth in musica instituyrthe vnd vntherweisethe, dergleichen auch die obgedachte kyrchen zw S. Mariemagdalenen mit gesange vnd anderem richtig hilde vnd also beyde kyrchen gleichmeßigk mit figurirthem gesange gecziret vnd erhalthen mochten werden. ${ }^{38}$

Erklärtes Ziel der Kommission von Stadtrat und Pfarrern ist es also, in den Gottesdiensten Figuralmusik erklingen zu lassen. Dafür wiederum muss ein »gelehrter Musicus « eingestellt werden, der den Schülern die nötigen Grundlagen vermitteln kann. Als Vorbild wird die Schule und Kirche von St. Elisabeth genannt, an der Virgilius Haugk die Pflege der Mensuralmusik bereits I54O offenbar so verwirklichte, wie es nunmehr auch für die Maria Magdalenen-Schule wünschenswert war. ${ }^{39}$ Haugk erfüllte also - obwohl offiziell nur als »Signator « bezeichnet - die Anforderungen, die an einen Cantor, also einen »gelehrten Musicus« gestellt wurden. ${ }^{40}$

35 Bauch, Aktenstücke (s. Anm. 34), hier S. 43.

36 Dass diese Unterscheidung um 1540 noch nicht ganz trennscharf war, zeigt das Ergebnis von Moibans Verhandlungen mit dem Stadtrat: Dort ist festgelegt, dass man für ein choraliter gesungenes Te Deum in einem Hochzeitsgottesdienst 24 Groschen zu bezahlen habe, von denen »dem cantori« sechs Groschen zustehen. Ein »mit der orgell gefigurirth[es] « Te Deum ist doppelt so teuer, »dem cantori« sind 48 Groschen zu bezahlen. Bauch, Aktenstücke zur Geschichte des Breslauer Schulwesens im XVI. Jahrhundert (s. Anm. 34). S. 44.

37 Niemöller, Untersuchungen (s. Anm. 3), hier S. 7f., außerdem Bauch, Geschichte (s. Anm. 3), hier S. 340 .

38 Bauch, Aktenstïcke (s. Anm. 34), hier S. 44.

39 Niemöller, Untersuchungen (s. Anm. 3), hier S. 9.

40 Gustav Bauch zählt Haugk »zu den Signatoren alten Stils [...], [der] eigentlich Kantor zu nennen wäre, da er ein >Musicus« war«; siehe: Geschichte (s. Anm. 3), hier S. 342. 
Somit bekleidete Virgilius Haugk das Signatorenamt in einer interessanten Umbruchszeit: Das Aufgabengebiet des Schulmusikers wurde größer - es schloss nunmehr auch die Figuralmusik ein - und damit der Anspruch an den schulischen Musikunterricht höher. Treibende Kraft für die Veränderungen war der humanistisch ausgebildete Pastor Ambrosius Moiban.

$I V$.

Aufschlüsse zu Virgilius Haugks Musikunterricht liefert sein bereits erwähntes Lehrbuch Erotemata musicae practicae ad captum puerilem formata (Fragen der praktischen Musik formuliert für den Verstand der Knaben). ${ }^{41}$ Es wurde im März I54I in der Breslauer Offizin des Andreas Winkler gedruckt. Am Ende des Werkes steht eine Elegie, die an den Breslauer Ratsherrn Sebastian Hennemann gerichtet ist, der als Vertreter des Rates I54O über Moibans Memorialzettel mit verhandelt hatte.

Bereits 1545 erlebte das Werk in Breslau eine zweite Auflage. ${ }^{42}$ Sie ist die Grundlage, auf der bisher alle Publikationen zu Haugk und seinen Erotemata entstanden. Häufig wird überhaupt nur die zweite Auflage erwähnt, man findet sogar die Behauptung, die erste Auflage des Druckes sei verloren. ${ }^{43}$ Tatsächlich existiert jedoch ein Exemplar davon in der Universitätsbibliothek Wrocław. ${ }^{44}$

4I Beschreibungen des Werkes sind zu finden bei Niemöller, Untersuchungen (s. Anm. 3), hier S. 9f; Lothar Hoffmann-Erbrecht und Fritz Feldmann, Musik in Schlesien, Dülmen I970, hier S. 35f.; Feldmann, »Studien zur Geschichte der Schulmusik Schlesiens« (s. Anm. 22), hier S. I69f., sowie bei Wilson, Choral pedagogy (s. Anm. 4), hier S. 538f. Siehe außerdem Klaus Wolfgang Niemöller: "Deutsche Musiktheorie im I6. Jahrhundert: Geistes- und institutionsgeschichtliche Grundlagen", in: Deutsche Musiktheorie des Is. bis I7. Jahrhunderts. Erster Teil: Von Paumann bis Calvisius, hrsg. von Theodor Göllner, Klaus Wolfgang Niemöller und Heinz von Loesch, Darmstadt 2003 (Geschichte der Musiktheorie 8/I), S. 69-98, hier S. 89 und 93.

42 Von dieser zweiten Auflage liegt heute ein Exemplar in der Bibliothèque Royale in Brüssel; siehe: Catalogue de la bibliothèque de F. J. Fétis acquise par l'état Belge, Nachdruck der Ausgabe Brüssel I877, Bologna 1969 (Bibliotheca musica Bononiensis I, 7), Nr. 5459, und eines in der Bibliothek des Prager Stiftes Strahov; vgl. Rudolf Quoika, »Christoph Harant von Poschitz und seine Zeit. Ein Kapitel der böhmischen Musikgeschichte der Renaissance«, in: Die Musikforschung 7 (1954), S. 4I4-429, hier S. 4I6. Die Signatur in Strahov lautet FP VI 9/a, freundliche Auskunft von Jan Pišna.

43 Birkendorf: Art. »Haugk, Virgilius« (s. Anm. 3).

44 Die Signatur lautet 380605 (freundliche Auskunft von Diana Lancucka). Von dem Breslauer Exemplar wiederum gibt es einen Mikrofilm in der Bayerischen Staatsbibliothek in München (Signatur: Film R 200I.28I,BUWrB-0386). Verzeichnet ist die Ausgabe von I54I bei Emil Bohn, Bibliographie der Musikdruckwerke bis 1700, welche in der Stadtbibliothek, der Bibliothek des Academischen Instituts für Kirchenmusik und der Königlichen und Universitäts-Bibliothek zu Breslau aufbewahrt werden. Ein Beitrag zur Geschichte der Musik im XV., XVI. und XVII. Jahrhundert, Nachdruck der Ausgabe Berlin I883, Hildesheim u.a. I969, hier S. II. Zur Geschichte der Breslauer Bibliotheksbestände siehe Aniela Kolbuszewska: »Historische Grundlagen der Musiksammlungen in der 
Die Publikation von Haugks Erotemata fügt sich nahtlos in das Bild von der Reform des Breslauer Schulwesens ein. Eine entscheidende Figur neben Ambrosius Moiban war der Buchdrucker Andreas Winkler, der seit Mitte der I520er Jahre das Rektorenamt an der Elisabeth-Schule bekleidete. Beide hatten pädagogische Erfahrungen vorzuweisen und außerdem eine Ausbildung an der Wittenberger Universität genossen. ${ }^{45}$ Bereits während seiner Breslauer Lehrtätigkeit zwischen I5I7 und I522 hatte sich Moiban darum bemüht, Lehrbücher herauszugeben, die für den Unterricht an einer Lateinschule geeignet waren und sich in der Präsentation des Stoffes wie in der sprachlichen Gestaltung an den Bedürfnissen der Schüler orientierten. ${ }^{46}$ Mit der Eröffnung von Winklers Breslauer Offizin I539 konnte Moiban die Verbreitung der Schulbücher weiter vorantreiben, nun unter vereinfachten Bedingungen: Der Rat der Stadt Breslau hatte Winkler die Errichtung der Druckerei Ende I538 genehmigt, damit der Mangel an Schulbüchern behoben werden könne und zu diesem Zweck auch einen Zuschuss gewährt. ${ }^{47}$ Haugks Erotemata wurden zwei Jahre nach der Eröffnung von Winklers Druckerei publiziert, man darf also davon ausgehen, dass sich das Werk in Moibans und Winklers humanistisch-reformatorisches Konzept einfügt. Bereits im Titel formuliert Haugk ja den von Moiban vertretenen Anspruch, sein Lehrbuch dem Verstand der Knaben anzupassen: ad captum puerilem formata.

Haugks Erotemata erweisen sich allerdings in mehrerer Hinsicht als etwas eigenwillig: Sie sind in Dialogform geschrieben (erotematische Methode), aber völlig ohne jede Kapiteleinteilung. ${ }^{48}$ Der Zugriff auf die Grundlagen der Musik

Universitätsbibliothek zu Breslau «, in: Die Musik der Deutschen im Osten und ibre Wechselwirkung mit den Nachbarn. Ostseeraum - Schlesien - Böhmen/Mähren - Donauraum, Kongressbericht Köln I992, hrsg. von Klaus Wolfgang Niemöller und Helmut Loos, Bonn I994 (Deutsche Musik im Osten 6), S. 295-302.

$45 \mathrm{Zu}$ Winklers Biographie siehe Adolf Schimmelpfennig: Art. »Winckler, Andreas«, in: Allgemeine Deutsche Biographie, Bd. 43, Berlin I898, S. 364-365, zu Winklers Druckerei außerdem Christoph Reske, Die Buchdrucker des I6. und I7. Jahrhunderts im deutschen Sprachgebiet, Wiesbaden 2007 (Beiträge zum Buch-und Bibliothekswesen 5I), hier S. I28. Die Angaben widersprechen sich zum Teil: Nach einem Studium in Krakau (Reske) oder in Wittenberg (Schimmelpfennig) wirkte Winkler ab I520 als Lehrer in Breslau und wurde I523 (Reske) bzw. I525 (Schimmelpfennig) zum Rektor der Elisabeth-Schule berufen. I535 erwarb er bei Melanchthon in Wittenberg den Magistergrad.

$\mathrm{Zu}$ Moibans Biographie siehe Dietrich Meyer, "Ambrosius Moiban (I494-I554) ", in: Schlesische Lebensbilder 9 (2007), S. IO9-I2O, außerdem Adolf Schimmelpfennig, Art. »Moibanus, Ambrosius", in: Allgemeine Deutsche Biographie, Bd. 22, Berlin I885, S. 8I-82. Auch in diesem Fall sind die biographischen Daten der beiden Autoren nicht völlig zur Deckung zu bringen: Moiban erwarb I5I4 das Baccalaureat in Krakau und I5I7 den Magistergrad in Wien. Er wurde I5I8 Rektor der Breslauer Domschule (Schimmelpfennig) bzw. I5I7 Lehrer daselbst, um I520 nach einem kurzen Aufenthalt in Wittenberg zum Rektor der Maria Magdalenen-Schule berufen zu werden. I522 begann er ein Theologiestudium in Wittenberg und kehrte I525 als Pastor der Elisabethkirche nach Breslau zurück.

46 Meyer, »Ambrosius Moiban (I494-I554)« (s. Anm. 45), hier S. IIO.

47 Reske, Buchdrucker (s. Anm. 45), hier S. I28.

48 Zur erotematischen Lehrmethode siehe z.B. Wilson, Choral pedagogy (s. Anm. 4), hier S. 69-7I. 
erfolgt in folgenden Schritten: Zunächst werden kurz die Grundlagen des einstimmigen Gesanges behandelt, dann in aller Ausführlichkeit und im Detail die Mensuralnotation, schließlich die Intervalle, die Kirchentonarten und dann erst die Mutation. ${ }^{49}$ Fritz Feldmann folgert aus dieser Gliederung, die Vermittlung der Mensuralmusik sei das pädagogische Hauptanliegen Haugks.

$\mathrm{Zu}$ seinen zentralen Absichten äußert sich Virgilius Haugk im Vorwort der Erotemata (BN 002 355-005 358). ${ }^{50}$ Das Vorwort richtet sich an die »studiosa iuventus « und benennt als ersten Zweck des Buches, ebendiese wissbegierige Jugend von der lästigen Abschreibearbeit im Unterricht zu befreien (»ut molestissimo aliquando describendi labore levaveris«, BN 002 355). Im folgenden appelliert Haugk an die Sorgfalt und den Eifer der Schüler (»ex tua diligentia sedulitateque «), als deren Frucht er ihnen Freude und Ehrbarkeit verspricht (»fructum laboris longe iucundissimum, voluptatem cum honestate iunctam«). Darauf setzt er zu einem allgemeinen Lob der Musik an (»Quid enim suavius, quam iucundissimis cantionibus levare miserias, labores, aerumnas? «, $\mathrm{BN} 003$ 356), das er relativ unvermittelt mit einem Verweis auf die Kürze seines Buches abbricht (»neque etiam libelli huius caperet angustia $\ll$ ).

Nun zeigt sich erstmals Haugks Polemik gegen altmodische Cantoren (»quosdam supina adeo stultitia praeditos «), denen er die musikalische Kompetenz abspricht (»sese non huius artis tantum sciolos«) und die er als Schmarotzer, Schauspieler und Narren bezeichnet (»sed et parasitos potius ac ludiones, et quod turpius est, etiam moriones quasi exhibere «).

Daraufhin kehrt Haugk wieder zu den Schülern zurück und betont, er habe sein Buch an deren Verstand ausgerichtet, und zwar sowohl inhaltlich als auch sprachlich (»me in hoc libello tuo accomodavi captui, idque non modo ipsa quam tibi trado materia, sed et dicendi modo«). Außerdem unterstreicht er noch einmal die Kürze seines Buches (»esse docendo cum prespicuitate brevem«, BN 004 357) und beruft sich dabei auf Horaz (»Quicquid praecipies esto brevis, ut cito dicta / Percipiant animi dociles, teneantque fideles $\ll) .{ }^{51}$

Haugk erklärt, sein Buch orientiere sich an den drei Kategorien, die ein Anfänger der Musik beherrschen muss, nämlich Tonhöhen, Noten(werte) und Intervalle (»huius artis tyroni tria esse observanda [...]. Nempe Clavium, Notarum, Intervallorum seu systematis rationem《). Auf dieser Grundlage habe er aus den zwei Arten der Musik (gemeint sind einstimmiger Choral und mehrstimmige Mensuralmusik) eine einzige und zusammenhängende gemacht (»ut e duabus musicis, unam atque perpetuam fecerim $\ll$ ).

49 Zusammenfassung nach Feldmann, »Studien« (s. Anm. 22), hier S. I70.

50 Der Druck der Erotemata weist keine Paginierung auf, deswegen werden hier als Referenz die Bildnummern (BN) des Münchener Mikrofilmes angegeben. Jede der Bildnummern bezieht sich auf eine Doppelseite. Bei allen Zitaten wurde die Schreibweise standardisiert.

5 I Das Zitat entstammt der Ars poetica des Horaz (CCCXXXV). 
Haugk argumentiert, dass sowohl die Mensuralmusik als auch der Choralgesang ihren jeweiligen Noten folgen (»Quid enim aliud Musica (ut vocant) mensuralis, quam variarum notularum mensuram prosequitur: An non \& plana ipsa seu choralis, suas quoque habet notulas? «). Er betrachtet die Noten also als das verbindende Element zwischen Choralgesang und Mensuralmusik und macht sie deshalb auch zum zentralen Gegenstand seines Traktates (»tota ipsa tractatio circa notulas versatur«). Die gängige Trennung von musica plana und musica mensuralis schreibt Haugk dem Eifer der Musikgelehrten zu (»Ex industria certe Musici rem alioqui facilem difficilem reddidere, cum e Musica una, duas atque etiam diversa fecere, in quibus tamen diversitatis est fere nihil «).

Auf das Einfügen von Beispielen und Übungen verzichtet Haugk, weil sie für die Schüler überall leicht zu finden seien und es viele zeitgenössische Komponisten gebe ("partim ob id quod nullibi fere non eiusmodi adunde reperiantur exercitia, partim quod nostra haec aetas harmonistas fere plures habeat quam Musicae studiosos $\ll$ ).

Am Ende des Vorwortes steht ein jambisches Schmähgedicht, das die Polemik Haugks gegen die altmodischen Cantoren wieder aufgreift und auf die Spitze treibt (BN o05 358):

Cæterum si quis fuerit, qui haec mea fastidierit, is sibi hisce Iambus responsum putet.

Nolo mem legas libellum, rancide

Cantor: tibi serena vox non est, neque

Serena mens, recede, displicient scio

Tibi, placent quae caeteris peroptime.

Nil dente livido nocebis pessime.

Non est tuum vereatur ut morsum, cui

Syncerus est animus, voluntas maxima

Omnes iuvare neminemque laedere.

Molestus esse si voles nobis, scias

Nos ista non tibi dedisse, sed bonis

Pueris, quibus pergrata speramus fore

Haec nostra. Abi, recede, cantor rancide.

Die Lektüre des Vorwortes und der Erotemata wirft zwei Fragen auf. Erstens: Gegen wen richtet sich die scharfe Polemik, wer ist der rückwärtsgewandte, altmodische Cantor? Zweitens: Hat sich Virgilius Haugk beim Verfassen seiner Erotemata an einem Vorbild orientiert?

Einen Anhaltspunkt bietet Haugks Vorhaben, den Choralgesang und die Figuralmusik in einer einzigen musica zusammenzufassen. Denn eine ähnliche Idee 
findet sich in den drei musiktheoretischen Werken des Sebald Heyden (I499-I56I), der zwischen I525 und I56I Rektor der Nürnberger St. Sebaldus-Schule war. ${ }^{52}$ In

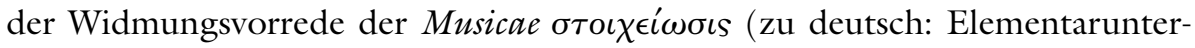
richt der Musik) wendet sich Heyden dezidiert gegen den gregorianischen Choral und dessen übermäßige Verwendung im Gottesdienst. ${ }^{53}$ Er betont den negativen Einfluss auf die Schüler, wenn diese nach dem mühsamen Unterricht in den Gottesdiensten die Choralmelodien singen müssen, und das nicht zu ihrer Erbauung oder zu ihrem eigenen Nutzen. Für die Knaben bedeute das Singen der Choralmelodien also nur Mühe und Last, vom Geist und der Wahrheit des Gottesdienstes trenne sie das Choralsingen eher, als dass es sie ihnen näher bringe.

$\mathrm{Zu}$ Beginn des eigentlichen Traktats erklärt Heyden dann, warum er die Choralnotation in seiner Abhandlung nicht berücksichtigt:

MUSICAE species sunt duae, Simplex et figurata. Simplex est, in qua omnes notulae, ut eiusdem fere formae ac coloris sunt, ita et quantitate aequivalent. Vulgus Choralem vocat. Ea vero, quia nihil plane habeat, quod non idem et figuratae sit, a me hic consulto omittitur. ${ }^{54}$

Heyden vertritt die Meinung, dass die Choralnotation in der Mensuralnotation enthalten ist und dass man demzufolge kein eigenes Studium der Choralnotation brauche. Deswegen gibt es in seiner Musicae $\sigma \tau o \iota \chi \epsilon i \omega \sigma \iota s$ auch kein Kapitel zur Choralnotation. Offensichtlich ist die Argumentation Virgilius Haugks für die Zusammenfassung der musica plana und der musica mensuralis also derjenigen Sebald Heydens ähnlich: Beide Autoren gehen davon aus, dass das Studium der Mensuralnotation für die Schüler völlig ausreichend ist, um auch die Choralmelodien singen zu können.

In diesem Punkt unterscheiden sich Haugk und Heyden deutlich von anderen reformatorisch beeinflussten Lehrwerken für den schulischen Musikunterricht, wie etwa von den Schriften Georg Rhaws und Nikolaus Listenius. ${ }^{55}$

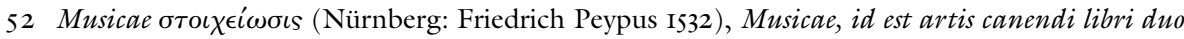
(Nürnberg: Johannes Petreius I537) und De arte canendi ... libri duo (Nürnberg: Johannes Petreius

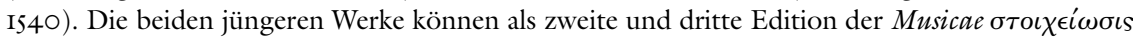
aufgefasst werden, die vor allem um zahlreiche Notenbeispiele erweitert wurden. Siehe dazu Clement A. Miller, "Sebald Heyden's De arte canendi: Background and contents«, in: Musica disciplina 24 (1970), S. 79-99, hier S. 79.

53 Eine deutsche Paraphrase dieser Vorrede bietet Alfred Kosel, Sebald Heyden (I499 - I56I). Ein Beitrag zur Geschichte der Nürnberger Schulmusik in der Reformationszeit, Würzburg 1940 (Literaturbistorisch-musikwissenschaftliche Abhandlungen 7), hier S. 27-29.

54 Fol. $6^{\mathrm{v}}-7^{\mathrm{r}}$. Zitiert nach dem Digitalisat der Bayerischen Staatsbibliothek München (URN: urn:nbn:de:bvb:12-bsb10187580-6, aufgerufen am I4.07.2016).

55 Die Unterscheidung zwischen Musiklehrbüchern für die Schule und solchen für die Universität nimmt Klaus Wolfgang Niemöller vor; siehe: Niemöller, »Deutsche Musiktheorie (s. Anm. 4I), hier S. 79-85 (universitäre Lehrbücher) und S. 89-92 (schulische Lehrbücher). Georg Rhaw: 
Ein weiteres Indiz weist darauf hin, dass Haugk Heydens Lehrwerk gekannt und

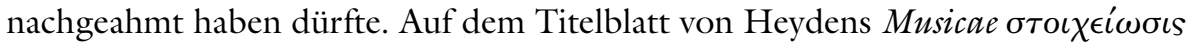
findet sich ein vierzeiliges Gedicht im elegischen Distichon, in dem sich der Autor an den kleinlichen Kritiker wendet:

\section{AUTOR ZOILO.}

Heus tu, ne dentes acuas, tibi Zoile dico.

Non tibi, sed pueris haec damus ingenius.

Hinc pudeat, moneo, rosisse aliena, quod ipsum

Stulticia est, ni des his meliora prius.

Ähnlich wie Haugk scheint auch schon Heyden Kritik an seinem Werk von vornherein einkalkuliert zu haben. Und so erklärt auch er (allerdings in weniger scharfem Ton als Haugk), sein Werk richte sich an die anständige Jugend und nicht an den hämischen Kritiker.

Es gibt also zusammenfassend zwei Gemeinsamkeiten der beiden Lehrbücher,

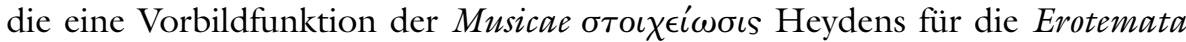
Haugks wahrscheinlich machen: Beide Autoren vertreten die Ansicht, das Studium der Mensuralmusik vermittle auch das nötige Wissen für das Singen von Choralmelodien und beide weisen mögliche Kritiker von vornherein in ihre Schranken. ${ }^{56}$

Wegen der mehrfach erwähnten Polemik des Vorwortes von Haugks Erotemata bietet es sich an, nach einem Negativvorbild zu suchen, von dem Haugk sich absetzen wollte - und damit möglicherweise der Frage näherzukommen, gegen wen die Polemik gerichtet ist.

Ein theologischer Gegner des Pastors Ambrosius Moiban und entschiedener Vertreter des alten Glaubens ist an dieser Stelle womöglich ein Schlüssel: Johannes Cochlaeus (I479-I552). Moiban hatte I533 einen lateinischen Katechismus verfasst, der 1535 in auch in deutscher Sprache erschien. ${ }^{57} \mathrm{Zu}$ diesem Katechismus veröffentlichte Cochlaeus I537 eine Gegendarstellung mit dem Titel Ein Kurtzer Bericht auff D. Moibanus Catechismum, in deren Einleitungspassage er die Zu-

Enchiridion utriusque musicae practicae (Wittenberg: Georg Rhaw I5I7), Nicolaus Listenius: Musica (Wittenberg: Georg Rhaw I537). Diese beiden Werke erlebten zahlreiche Neuauflagen und erfreuten sich extrem weiter Verbreitung: Rhaws Enchiridion wurde allein in Wittenberg zehnmal nachgedruckt, Listenius' Musica über vierzigmal an verschiedenen Orten; vgl. Niemöller: »Deutsche Musiktheorie« (s. Anm. 4I), hier S. 74f.

56 Eine genaue Überprüfung, inwieweit sich die Lerninhalte und die Lehrmethode in den Traktaten

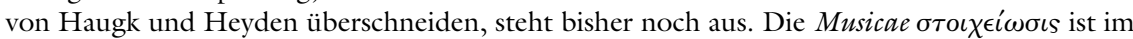
Unterschied zu den Erotemata jedenfalls nicht in Dialogform geschrieben.

57 Ambrosius Moiban, Catechismi Christiani Capita, Iuvenibus his, qui in literis honestis, progressum aliquem fecerunt proponenda, Wittenberg: Hans Weiß I533; Ambrosius Moiban, Catechismus, Auff zehen Artickel Götlicher schrifft gestellet wie man fur Gott vnd den menschen ein Christlich frumes leben furen sol, Wittenberg: Hans Weiß I535. 
stände an den Breslauer Pfarrkirchen kritisiert: ${ }^{58}$ Zunächst betont Cochlaeus, er kenne Moiban nicht persönlich und wolle ihn auch nicht persönlich angreifen, vielmehr gehe es um die Religion und die Tatsache, dass »ers mit dem Luther helt « (BN 6-7). Cochlaeus kritisiert die beiden Breslauer Pastoren: Der eine Prediger (Ambrosius Moiban) sei ein Laie und habe daher kein Recht, ein Predigeroder Priesteramt auszufüllen, der andere (Johann Heß) sei zwar ein geweihter Priester, aber mittlerweile verheiratet ( $\mathrm{BN} 7-8)$. Cochlaeus ist es »warlich leyd for die feinen / mechtigen und hochberümpten Stat Breßlau « (BN IO), weil sie sich von den beiden für ihn nicht akzeptablen Pastoren vom rechten Glauben abbringen lasse. Nach einem Abschnitt über die Verdienste des alten Breslau für die katholische Kirche stellt Cochlaeus erneut die Berechtigung Moibans und Heß’ für ihre Ämter in Frage. Doch kommt Cochlaeus nicht umhin, auch seine Anerkennung für Moiban zu äußern: »so vil er von zucht unn tugent saget / laß ich mir wol gefallen / wo es unvermenget mit yrtumb ist $(\mathrm{BN} 20)$.

Ab 1539 hatte Cochlaeus ein Kanonikat am Breslauer Domkapitel inne und lebte von $\mathrm{I} 539$ bis $\mathrm{I} 543$ und von $\mathrm{I} 549$ bis zu seinem Tode $\mathrm{I} 552$ in Breslau. ${ }^{59}$ Kurz nach dem Verfassen seiner Schrift gegen Moiban und kurz vor der Veröffentlichung von Haugks Erotemata kam Cochlaeus also nach Breslau und bemühte sich dort, der »weiteren Protestantisierung des Bistums vorzubeugen. $"{ }^{60}$ Er wurde also zu einem direkten Gegenspieler Moibans, der seinerseits die Reformation in Breslau vorantrieb. Außerdem war Cochlaeus bestrebt, in Breslau eine Druckerei für katholisches Schriftgut einzurichten, ${ }^{61}$ was ihm nicht gelang - in seiner Zusammenarbeit mit Andreas Winkler war Moiban ihm auch auf diesem Gebiet ein Konkurrent. Diese kurze Skizze mag genügen, um eine Gegnerschaft von Moiban und Cochlaeus plausibel zu machen. Zieht man in Betracht, dass die Veröffentlichung von Haugks Erotemata I54I höchstwahrscheinlich unter dem Einfluss Moibans zustande kam, und dass Cochlaeus selbst ein musiktheoretisches Lateinschul-Lehrbuch verfasst hat, so lohnt es sich, über mögliche Beziehungen von Haugks Erotemata und Cochlaeus' Tetrachordum musices (Nürnberg: Johann Weyssenburger I5II) nachzudenken.

58 Johannes Cochlaeus, Ein Kurtzer Bericht auff D. Moibanus Catechismum, Leipzig: Nikolaus Wolrab I537, zitiert nach dem Digitalisat der Bayerischen Staatsbibliothek München (URN: urn:nbn:de:bvb:12-bsb11119119-9, aufgerufen am I4.07.2016). Die Angaben der Bildnummern (BN) im Text beziehen sich auf dieses Digitalisat.

59 Remigius Bäumer, Johannes Cochlaeus (1479-I552). Leben und Werk im Dienst der katholischen Reform. Münster 1980 (Katholisches Leben und Kirchenreform im Zeitalter der Glaubensspaltung 40). Zu Cochlaeus' Breslauer Jahren dort v. a. S. 5If. und $65 f$.

60 Ebenda, S. 5 I.

6 I Ebenda, S. 52. Cochlaeus fand tatsächlich einen Verleger für seine Ansprüche (wenn auch nicht in Breslau), den von Leipzig nach Mainz übergesiedelten Franz Behem. 
Cochlaeus schrieb das Tetrachordum in seiner Zeit als Rektor der St. LorenzSchule in Nürnberg (I5IO-I5I5). Es ist in vier Traktate gegliedert: De musicis elementis, De Musica Gregoriana, De octo tonis meli und De musica mensurali. Die drei Hauptanliegen seines Werkes nennt Cochlaeus in der Vorrede an Anton Kreß, den Propst von St. Lorenz: die fromme und gründliche Erziehung der Jugend (»ut sancta ac pervigil esset iuventutis nostrae / et in litteris / et in moribus eruditio«), die korrekte tägliche Ausführung des Choralgesangs (»ut quotidianus ille ecclesiasticus cantus / diligenter ac integre absolveretur«) sowie die Pflege der Mensuralmusik (»ut figurabilem quoque concentum (quo populus noster non mediocriter oblectatur) non negligerem omnino $«) .^{62}$

Gegenüber älteren Musiktraktaten für den schulischen Gebrauch erweiterte Cochlaeus im Tetrachordum die Chorallehre um mehrere Aspekte, doch das entscheidende Novum ist die Berücksichtung der Mensuralmusik in einem Lateinschullehrbuch. ${ }^{63}$ Es ist höchst fraglich, ob Virgilius Haugk sich der Tatsache bewusst war, dass Cochlaeus der erste Autor war, der in einem Schullehrbuch sowohl Choral- als auch Figuralmusik behandelte. Doch kann Cochlaeus Haugk als exponierter Vertreter der von ihm kritisierten und für überflüssig befundenen Zweiteilung der Musik gegolten haben, da das Tetrachordum weit verbreitet und bekannt war. ${ }^{64}$

Als Fazit lässt sich festhalten: Die Erotemata musicae practicae ad captum puerilem formata des Virgilius Haugk können als Beleg für einen »Kleinmeister« auf dem Gebiet des Musikunterrichts gelten. Haugks Traktat erscheint weniger gut verständlich und nicht so übersichtlich gegliedert wie die Werke seiner bekannteren Zeitgenossen, doch vermag es einen willkommenen Einblick in den Breslauer Musikunterricht um I540 zu gewähren.

\section{$V$.}

Schließlich sollen auch die Kompositionen von Virgilius Haugk kurz betrachtet werden. Als Beispiel soll eine der Responsorienvertonungen aus der Zwickauer Schalreuter-Handschrift dienen. Wie eingangs bereits beschrieben, sind dort die vier Responsorien Verbum caro factum est à 4 (Weihnachten), Ite in orbem uni-

62 Zitiert nach dem Digitalisat der Bayerischen Staatsbibliothek München (URN: urn:nbn:de:bvb:12 bsb00007447-9, aufgerufen am I4.07.2016).

$63 »[B]$ ei den Kirchentönen [werden] nicht nur die antiphonale Psalmodie, sondern auch die Introiten und Responsorien ausführlich behandelt, und liturgisch-musikalische Erläuterungen über die verschiedenen Formen des gregorianischen Gesangs sowie des smodus legendi choraliter [gegeben].« Niemöller, Untersuchungen (s. Anm. 3), hier S. 338.

64 Auch der Zusammenhang zwischen Haugks Erotemata und Cochlaeus' Tetrachordum bedarf noch genauerer Untersuchung. Zu prüfen wäre außerdem, ob Sebald Heyden, der ja ein CochlaeusSchüler war, sich bewusst von seinem Lehrer abwandte. 
versum à 5 (Christi Himmelfahrt), Apparuerunt apostolis à 5 (Pfingsten) und In monte oliveti à 5 (Gründonnerstag) überliefert. ${ }^{65}$

Weil Haugk jeder Vertonung den entsprechenden gregorianischen Choral zugrunde legt, ist es sinnvoll, sich zunächst Klarheit über die Struktur der einstimmigen Responsorien zu verschaffen: Sie haben einen zwei- bzw. dreiteiligen Aufbau in Caput, Repetenda (beides zusammen wird auch als Corpus bezeichnet) und Versus ${ }^{66}$ Für die Vortragsweise sind zwei Dinge charakteristisch: erstens die Refrainform, die durch die Wiederholung der Repetenda entsteht, und zweitens der Wechsel zwischen solistischer und chorischer Besetzung. Insgesamt ergibt sich also folgendes Schema:

Caput (Solist/Chor) - Repetenda (Chor) - Versus (Solist) - Repetenda (Chor)

Die vier Responsorien von Virgilius Haugk ordnen jedem der Responsorienteile eine Motettenpars zu, sie sind sämtlich dem additiven Formprinzip zuzuordnen. ${ }^{67}$ Das ist durchaus typisch für die Responsorien deutscher Komponisten in der ersten Hälfte des I6. Jahrhunderts. Diese dreiteiligen Responsorienvertonungen haben in der Regel einen starken Bezug zur liturgischen Praxis und stammen meist von Komponisten, die im deutschsprachigen Raum an einer Hofkapelle oder Lateinschule tätig waren, sprich: für die musikalische Gestaltung der Gottesdienste zu sorgen hatten. ${ }^{68}$ Es ist also sehr wahrscheinlich, dass diese Stücke tatsächlich im liturgischen Gebrauch waren und die einstimmigen Responsorien ersetzten.

Die Tatsache, dass Haugks Responsorien in einer Liebhaber-Handschrift überliefert sind, die definitiv nicht für den liturgischen Gebrauch gedacht war, bedeutet dabei nicht, dass die Stücke in Breslau nicht liturgisch korrekt im Gottesdienst gesungen wurden. Doch ist sie ein Beleg für den paraliturgischen Einsatz der Responsorien (d.h. ihre Aufführung zwar im Gottesdienst, aber ohne die eigentliche liturgische Funktion) sowie für die Verwendung als Spruchmotetten, die vielleicht im privaten Kreis gesungen wurden. ${ }^{69}$

Schließlich soll versucht werden, Haugks Kompositionsstil etwas differenzierter zu beschreiben: Als Einstieg soll die oben formulierte These aufgegriffen werden, dass Haugks Kompositionen im Kontext von Georg Rhaws Wittenberger Sammlungen zu verorten sind.

65 Die Stücke finden sich in der Edition Die Handschrift des Jodocus Schalreuter (s. Anm. IO). Apparuerunt Apostolis: Bd. 3, S. 22-26, die anderen drei in Bd. 4: Ite in orbem S. 24-28, Apparuerunt Apostolis S. 28-32 und In monte oliveti S. 130-I36.

66 Siehe dazu Schwemer, Mehrstimmige Responsorienvertonungen (s. Anm. IO), hier Bd. I, S. IO-I4; David Hiley, Helmut Hucke, Art. »Responsorium«, in: $M G G^{2}$, Sachteil Bd. 8, Kassel u.a. 1998, Sp. I76-200, hier Sp. I79-I8I und I93-199.

67 Schwemer, Mehrstimmige Responsorienvertonungen (s. Anm. IO), hier Bd. I, S. 42-44.

68 Ebenda, S. 193-I95.

69 Ebenda, S. 203-205 
Als Vergleichsmaterial zu Haugks Responsorien bietet sich Rhaws Individualdruck der Responsorienvertonungen des Balthasar Resinarius an. Es handelt sich dabei um zwei Bände mit dem Titel Responsoriorum numero octoginta ... (RISM R II96), die Rhaw I543 druckte. ${ }^{70}$ Wie schon Rhaws Sacrorum bymnorum liber primus von 1542 ist auch der Responsoriendruck zweigeteilt, in ein Temporale und ein Sanctorale. Und ebenso wie dieser gehören die beiden Resinarius-Bände zu Rhaws Drucken mit Musik für den evangelischen Vespergottesdienst.

Von den vier Responsorientexten, die Haugk vertonte, finden sich drei auch in der Sammlung von Balthasar Resinarius, so dass sich ein direkter Vergleich anbietet. Ein solcher Vergleich der Bearbeitungen kann aber lediglich dazu dienen, eine stilistische Verwandtschaft zu überprüfen, er kann keine Abhängigkeit oder Einflüsse nachweisen. Denn es ist nicht klar, welche Responsorienbearbeitungen die älteren sind. Haugks Responsorien sind undatiert überliefert, es ist allenfalls wahrscheinlich, dass er sie in seiner Breslauer Amtszeit als Signator schrieb (I538-I544). Die Resinarius-Responsorien sind zwar 1543 gedruckt, aber es ist nicht ausgeschlossen, dass sie schon früher komponiert wurden. ${ }^{71}$

Dem Resinarius-Druck sind zwei Vorworte vorangestellt, eines von Johannes Bugenhagen und eines von Georg Rhaw. Dass in den beiden Vorworten eine Kontroverse der Verfasser über die Heiligenresponsorien aufbricht, sei hier nur am Rande erwähnt. ${ }^{72}$ Rhaw unterstreicht jedenfalls die Bedeutung und die melodischen Qualitäten der alten Choralmelodien, um damit die Beibehaltung der Heiligenresponsorien zu rechtfertigen. Während Bugenhagens Argumentation auf den theologisch einwandfreien Inhalt der Responsorientexte zielt, ist Rhaws auf die Choralmelodien und ihren Erhalt gerichtet.

Sowohl in den Responsorien von Resinarius als auch in denen von Haugk ist die Choralmelodie wichtiger Bestandteil des Satzes. Doch lassen sich auch deutliche stilistische Unterschiede feststellen. Beispielhaft sollen die beiden Vertonungen von Apparuerunt Apostolis, dem Responsorium für das Pfingstfest, nebeneinandergelegt werden. Der Responsorientext beruht auf zwei Versen der biblischen Pfingsterzählung (Apostelgeschichte 2,3-4) und lautet:

70 Es gibt eine moderne Ausgabe der Sammlung: Balthasar Resinarius, Responsoriorum numero octoginta, 2 Bände, hrsg. von Inge-Maria Schröder, Kassel 1955/I957. Für eine kurze Beschreibung des Rhaw-Druckes siehe Schwemer, Mehrstimmige Responsorienvertonungen (s. Anm. IO), hier Bd. I, S. 2If. und S. IO4-I26. Siehe auch die ältere Untersuchung von Inge-Maria Schröder, Die Responsorienvertonungen des Balthasar Resinarius, Kassel u.a. 1954 (Schriften des Landesinstituts für Musikforschung Kiel 2).

7 I Irmlind Capelle, Art. »Resinarius«, in: $M G G^{2}$, Personenteil Bd. I3, Kassel u.a. 2005, Sp. I564-I566, hier Sp. I566.

72 Siehe dazu Schwemer, Mehrstimmige Responsorienvertonungen in deutschen Quellen des I5. und I6. Jahrhunderts (s. Anm. IO), hier Bd. I, S. IO8-II3, außerdem Raimund Redeker, Lateinische Widmungsvorreden zu Mess- und Motettendrucken der ersten Hälfte des I6. Jahrhunderts, Eisenach I995 (Schriften zur Musikwissenschaft aus Münster 6), hier S. 259-26I und S. 3I5-325. 
Apparuerunt apostolis dispertitae linguae tamquam ignis alleluia

Seditque supra singulos eorum spiritus sanctus alleluia

Loquebantur variis linguis apostoli Magnalia dei.

Die Vertonungen von Haugk und Resinarius haben beide den gleichen dreiteiligen Aufbau mit zweigeteiltem Versus, sowie etwa den gleichen Umfang (siehe Tabelle I):

\begin{tabular}{lll} 
& Resinarius & Haugk \\
\hline Caput & $\mathbf{3 2}$ Mensuren & 49 Mensuren \\
& Schlusskadenz auf g & Schlusskadenz auf g \\
\hline Repetenda & 40 Mensuren & 40 Mensuren \\
& Schlusskadenz auf g & Schlusskadenz auf g \\
\hline Versus & $\mathbf{4 9}(\mathbf{2 I}+\mathbf{2 8})$ Mensuren & 30 (I6 + I4) Mensuren \\
& Schlusskadenz auf g & Schlusskadenz auf c \\
& geteilt durch Doppelstrich & geteilt durch Generalpause \\
& und Besetzung: & (Diskant, Altus vs. tutti) \\
\hline & (Altus, Tenor, Bassus vs. tutti) & (Disetzung: \\
& I2I Mensuren & II9 Mensuren
\end{tabular}

Tabelle I: Aufbau der Apparuerunt apostolis-Vertonungen von Haugk und Resinarius

Resinarius' Stil ist in der Literatur von Inge-Maria Schröder und Bettina Schwemer beschrieben worden. ${ }^{73}$ Sein wichtigstes Merkmal ist die strenge Orientierung an der Choralvorlage - damit entsprechen die Stücke dem Wunsch Georg Rhaws, die alten Choralmelodien zu erhalten.

In Resinarius' Vertonung von Apparuerunt Apostolis ist der Choral als Cantus prius factus stets präsent und liegt im Diskant (sonst meist im Tenor) ${ }^{74}$ Jeweils zu Beginn der Choralabschnitte imitieren die anderen Stimmen den C.p.f., ansonsten sind sie melodisch frei gestaltet. Resinarius strukturiert den C.p.f. durch Pausen und Kadenzen. Dabei erscheinen die Kadenzen ausschließlich an sprachlichsyntaktisch sinnvollen Stellen. Resinarius verwendet eine einfache, übersichtliche Satztechnik ohne komplexe Formen wie Kanons oder weitreichende Imitatio-

73 Schröder, Responsorienvertonungen (s. Anm. 70), hier S. 53-80; Schwemer, Mehrstimmige Responsorienvertonungen (s. Anm. IO), hier Bd. I, S. II5-I26.

74 Resinarius' Apparuerunt Apostolis steht in der Neuausgabe des Responsoriendruckes; siehe: Balthasar Resinarius, Responsoriorum numero octoginta (s. Anm. 70), Bd. I, S. II9-I22. Die Quelle für die Choralvorlage ist Responsoria noviter cum notis impressa: de tempore et sanctis per totum annum: regentibus et scolaribus vtilissima. [Nürnberg]: Georg Stuchs I509, fol. 47. Digitalisat der Universitätsbibliothek München (URN: urn:nbn:de:bvb:19-epub-11936-3, aufgerufen am I3.07.20I6). 
nen. Erwähnenswert ist schließlich die häufige Verwendung der Unterterz- oder Landino-Klausel, die im I6. Jahrhundert eigentlich nicht mehr im Gebrauch war und die den Responsorien einen archaischen und formelhaften Charakter verleiht. Oberstes Ziel der Vertonung - neben der konsequenten Verwendung des Chorals scheint die sinnvolle Textdisposition und damit die Textverständlichkeit zu sein.

Auch Virgilius Haugk orientiert sich eng an der Choralvorlage. ${ }^{75}$ Sein Satz von Apparuerunt Apostolis weist jedoch stilistische Unterscheide zu dem von Resinarius auf. Zunächst einmal ist Haugks Vertonung fünfstimmig, wodurch er mehr satztechnischen Spielraum hat als Resinarius.

Im Caput liegt der Choral meist im Diskant, wird aber von freien Einschüben unterbrochen (M. II-I3, 23-27, 33). Die jeweils recht kurzen Choralabschnitte sind zwar weitgehend in Semibreven rhythmisiert, doch fallen sie im Satz kaum als Choralmelodie auf, denn die anderen vier Stimmen werden durch Imitation an der Choralmelodie beteiligt - auch über die Anfangsimitation hinaus. Am Schluss des Caput liegt die Choralmelodie des Allelwia in Breven im Diskant und ist somit deutlich als solche erkennbar.

In der Repetenda ist der Tenor die choraltragende Stimme, sonst ist der Satz dem des Caput ähnlich. Der Versus ist durch die Besetzung und eine Generalpause zweigeteilt: Im ersten Teil singen nur Diskant und Altus, der C.p.f. liegt in Semibrevenbewegung und eine Quinte nach oben transponiert im Diskant. Der Altus ist im wesentlichen frei geführt, beginnt jedoch mit einer Vorausimitation. Im zweiten Teil liegt der C.p.f. wieder in Semibrevenbewegung im Tenor, wird von den anderen Stimmen jedoch nicht imitiert. Beendet wird der Versus mit einer authentischen Kadenz nach c. Da dies ein choralfremder Ton ist, liegt die Vermutung nahe, dass Haugk das Responsorium für einen liturgisch korrekten Vortrag mit Wiederholung der Repetenda komponiert hat, denn am Ende der Repetenda entspricht der Schlusston des Chorals dem Grundton des Schlussakkordes (g).

Folgende Stellen in den Responsorien stechen bei dem Vergleich zwischen Haugk und Resinarius besonders ins Auge: Auffällig ist die Vertonung des Wortes »ignis« im Caput der beiden Vertonungen. Resinarius versieht es mit einer klangvollen Sextparallele zwischen Tenor und Bassus (M. I9-2I), der eine Terzparallele zwischen Altus und Tenor vorausgeht (M. I8-I9).

Haugk komponiert zum Wort »ignis« erst eine klangvolle Dezimparallele zwischen Diskant und Bassus (M. 33), dann eine rauh klingende Sextakkordkette der

75 Im Kommentar der Edition der Schalreuter-Handschrift sind die Quellen für die Choralvorlage nachgewiesen. Die Handschrift des Jodocus Schalreuter (s. Anm. IO), hier Bd. 4, S. 250. Die identifizierten Quellen sind das Antiphonale pataviense, fol. I2I, moderne Edition: Antiphonale Pataviense (Wien I5I9), hrsg. von Karlheinz Schlager, Kassel u.a. 1985 (Das Erbe deutscher Musik 88), Digitalisat der Bayerischen Staatsbibliothek München (URN: urn:nbn:de:bvb:12-bsb00080050-0, aufgerufen am 13.07.2016) und der Responsoriendruck von Georg Stuchs (s. Anm. 74), fol. 47. 
drei Unterstimmen (M. 35-36). In beiden Fällen münden die parallelen Sexten in eine Kadenz auf den Choralton a.

Die Tenor- und Bassus-Stimmen von Resinarius und Haugk sind an dieser Stelle verblüffend ähnlich - beinahe identisch - gestaltet, bei Haugk vervollständigt der Quintus den kurzen Fauxbourdon-Satz.

Interessant ist es auch, wie die beiden Komponisten mit melodischen Wiederholungen umgehen, die die Choralmelodie vorgibt. In der Repetenda etwa enthält die Choralmelodie zu den direkt aufeinander folgenden Worten »eorum« und »spiritus « jeweils die Tonfolge d-c-h-a-c. Bei Resinarius nehmen die beiden Worte mit fünf Semibreven jeweils zweieinhalb Mensuren ein (M. 47-49 und 52-54). Diese zweieinhalb Mensuren sind in allen vier Stimmen identisch ausgesetzt. Haugk dagegen verkürzt die Tonfolge auf den Quartgang d-c-h-a und lässt ihn (auch in der transformierten Fassung g-f-e-d im Quintus) durch die Stimmen wandern (M. 70-76). Durch die versetzten Einsätze entstehen dabei klangvolle Terzparallelen.

Haugks analytischer Blick auch für derartige Strukturen des Chorals, die nicht sofort ins Auge stechen, zeigt sich im Versus: ${ }^{76}$ In dem langen Choralmelisma zu dem Text »magnalia dei « kommt die Tonfolge e-d-c-d vor, etwas später $\mathrm{c}-\mathrm{d}-\mathrm{e}-\mathrm{d}$ und nochmals e-d-c-d. Alle drei versieht Haugk mit der gleichen kadenzierenden Formel c-h-c-g in Diskant und Bassus (M. IO6-II5).

Der Vergleich führt zu folgendem Resultat: Die große Gemeinsamkeit der Responsorien von Resinarius und Haugk besteht in der stets dreiteiligen Form, die auf eine gewisse Nähe zur liturgischen Praxis schließen lässt. Beide orientieren sich bei den Responsorienvertonungen an der Choralvorlage und binden sie in den Satz ein. Doch tun sie das auf unterschiedliche Weise: Während bei Resinarius das Hauptaugenmerk auf der Textdisposition und -verständlichkeit liegt, ist Haugks Satz kontrapunktisch differenzierter gearbeitet.

\section{Fazit}

Welchen »Wert für die Geschichtsschreibung « hat also der »Kleinmeister« Virgilius Haugk zu bieten? Trotz der dürftigen Faktenlage zu seiner Biographie und der nicht gerade üppigen Überlieferungssituation seiner Werke weisen die erhaltenen Quellen auf eine - wenn auch indirekte - Beziehung Haugks zum Wittenberger Notendrucker Georg Rhaw hin. Einen Einblick in die liturgische Praxis in Breslau um I540 vor dem Hintergrund der Einführung der Reformation und der Neuordnung des Schul- und Kirchenwesens können die überlieferten Werke Haugks

76 Im zweiten Teil des Versus zum Text »magnalia Dei« verwenden Resinarius und Haugk interessanterweise nicht den gleichen C.p.f.: Resinarius' Choralmelodie entspricht der in Georg Stuchs' Responsoriendruck, Haugks dagegen derjenigen im Antiphonale pataviense. 
gewähren. Die in allen Stimmbüchern erhaltenen Stücke aus den Rhaw-Drucken und der Zwickauer Schalreuter-Handschrift können sogar einen hörbaren Eindruck davon wiedergeben. Der »Fall« Haugk ist also ein Beispiel dafür, wie das reformatorische Zentrum Wittenberg auf die Peripherie (in diesem Fall die schlesische) wirkte, aber auch für die Aufnahme von an der Peripherie entstandenen Werken in prominente Wittenberger Quellen.

Mit der Verwendung alter Choralmelodien hat sich Haugk nicht nur in seinen Kompositionen beschäftigt, sondern auch in seinen Erotemata musicae practicae. Dieses - ebenfalls periphere - Lehrwerk hat bisher in der Forschung wenig Beachtung gefunden. Seinen Inhalt und Kontext genauer zu untersuchen, könnte womöglich den Wert Haugks für die Musikgeschichtsschreibung noch steigern. 